\title{
Endosonografía con biopsia por aspiración con aguja fina de glándula suprarrenal izquierda para el diagnóstico de paracoccidioidomicosis: aporte de un nuevo caso a la literatura
}

\section{Endosonography with Fine Needle Aspiration Biopsy of the Left Adrenal Gland for Diagnosis of Paracoccidioidomycosis: Contribution of a New Case to the Literature}

\author{
Edgar Julián Ferreira B., ${ }^{1}$ Germán Manuel Tovar F., ${ }^{2}$ Jairo Mendoza S., ${ }^{3}$ María Emma García, ${ }^{4}$ Gustavo Adolfo Parra S., ${ }^{5}$
}

Edwin Antonio Wandurraga S. ${ }^{6}$

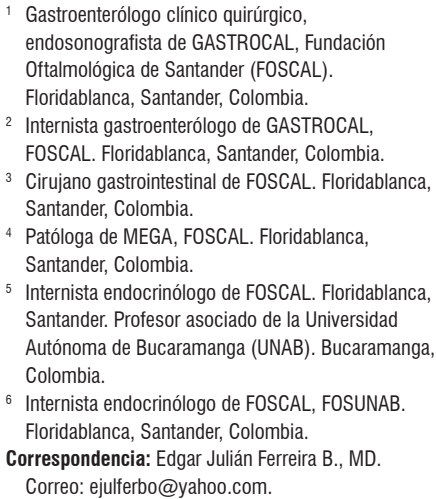

6 Internista endocrinólogo de FOSCAL, FOSUNAB.

Floridablanca, Santander, Colombia.

Correspondencia: Edgar Julián Ferreira B., MD. Correo: ejulferbo@yahoo.com.

\begin{abstract}
Resumen
La endosonografía nació hace más de 30 años como parte de las modalidades diagnósticas de patologías benignas y malignas del tracto gastrointestinal. En los últimos años se ha demostrado la eficacia y seguridad superior de la biopsia con aguja fina de las glándulas suprarrenales por vía transgástrica (izquierda) o tranduodenal (derecha) guiadas por endosonografía, en comparación con los abordajes percutáneos guiados por tomografía axial computarizada (TAC) o ecografía. Uno de los diagnósticos diferenciales de insuficiencia suprarrenal con evidencia de masas en glándulas suprarrenales es la paracoccidioidomicosis, a pesar de ser una entidad de presentación exclusiva de las áreas geográficas de Latinoamérica, donde se encuentra su agente etiológico. Se presenta un caso de paciente con paracoccidioidomicosis con insuficiencia suprarrenal y engrosamiento adrenal bilateral, cuyo diagnóstico se logró mediante biopsia transgástrica de glándula suprarrenal izquierda guiada por endosonografía.
\end{abstract}

Palabras clave

Endosonografía, paracoccidioidomicosis, insuficiencia suprarrenal (fuente: DeCS).

\begin{abstract}
Endosonography, born more than 30 years ago, is one diagnostic modality for benign and malignant pathologies of the gastrointestinal tract. In recent years, transgastric (left) or transduodenal (right) fine needle biopsies of the adrenal glands guided by endosonography has been demonstrated to be safer and more efficacious than percutaneous approaches guided by CT or ultrasound. Differential diagnoses of adrenal insufficiency with evidence of masses in adrenal glands must consider paracoccidioidomycosis despite the fact that it is exclusively present in Latin America where its etiological agent is found. We present a case of paracoccidioidomycosis with adrenal insufficiency and bilateral adrenal thickening whose diagnosis was achieved by transgastric biopsy of the left adrenal gland guided by endosonography.
\end{abstract}

Keywords

Endosonography, paracoccidioidomycosis, adrenal insufficiency (source: DeCS).

\section{INTRODUCCIÓN}

La ultrasonografía endoscópica (endosonografía) nació hace 30 años como parte de las modalidades diagnósticas de patologías benignas y malignas del tracto gastrointes- tinal. En la actualidad, se utiliza ampliamente con ese fin y desde hace más de una década también se utiliza con intención terapéutica con múltiples opciones (derivaciones biliodigestivas, abordajes combinados para la vía biliar con técnica de Rendez-vous, entre otras) principalmente en el 
tracto gastrointestinal superior (1). Los equipos de ultrasonografía endoscópica posibilitan tomar muestras de biopsia por punción transesofágica, transgástrica, transduodenal o transrectal, lo que permite un estudio más profundo y adecuado. Dicha posibilidad de obtener tejido por medio de biopsia con aguja también permite el diagnóstico diferencial de múltiples patologías tumorales e inflamatorias, y en estas últimas ayuda muchas veces a aclarar la etiología específica y, por tanto, establecer un tratamiento adecuado. En los últimos años se ha demostrado la eficacia y seguridad superiores de la biopsia de las glándulas suprarrenales por vía transgástrica o tranduodenal guiadas por ultrasonido endoscópico, en comparación con los abordajes percutáneos guiados por tomografía axial computarizada (TAC) o ecografía.

En la actualidad, se sabe que hasta el 5\% de todos los pacientes a los que se les realiza una TAC del abdomen tienen como hallazgo incidental la presencia de una masa adrenal, que en muchas ocasiones requiere de estudios complementarios para esclarecer el diagnóstico final (2). En los reportes de la literatura de biopsia de glándula suprarrenal guiada por endosonografía se ha demostrado la presencia de lesiones tumorales malignas metastásicas de múltiples órganos (pulmón, mama, estómago, riñón, piel y sistema linfático); lesiones benignas e infecciosas, predominantemente tuberculosis e histoplasmosis; y solo hasta años recientes se logró diagnosticar paracoccidioidomicosis mediante biopsia de la glándula suprarrenal izquierda guiada por endosonografía $(3,4)$. Por lo anterior, se decidió reportar un caso de diagnóstico de paracoccidioidomicosis a través de biopsia de glándula suprarrenal izquierda guiada por endosonografía.

\section{CASO CLÍNICO}

Paciente de género masculino de 56 años de edad, que consultó al servicio de urgencias por cuadro agudo de debilidad generalizada, astenia, adinamia y diaforesis; en su ingreso a urgencias se observó la presencia de hipotensión severa y deshidratación grado I. Como hallazgos adicionales al examen físico, se documentaron la presencia de hepatomegalia y lesiones en la piel del dorso de las manos y cara hiperpigmentadas y de bordes regulares. Como antecedentes de importancia, es agricultor procedente de Molagavita, área rural del Departamento de Santander (Colombia) y actualmente residente en el área urbana de Girón, Santander (Colombia). Como único antecedente patológico, es diabético compensado. Desde 8 meses antes de su consulta a urgencias venía presentando síntomas de astenia, adinamia y pérdida de aproximadamente $15 \mathrm{~kg}$ de peso, y un mes antes del cuadro agudo severo, requirió de un manejo intrahospitalario para la corrección de hiponatremia sintomática.
Se inició el manejo médico inmediato con hidratación intravenosa (IV) y en su estudio paraclínico llamó la atención la presencia de un reporte de cortisol sérico de las 8 a.m. en $0,0 \mu \mathrm{g} / \mathrm{dL}$, hiponatremia con valor de sodio sérico en $125 \mathrm{mmol} / \mathrm{L}$ e hiperpotasemia severa con potasio sérico en $7 \mathrm{mmol} / \mathrm{L}$ (sin alteraciones electrocardiográficas). Otros exámenes reportaron hiperfosfatemia con valor de $5,9 \mathrm{mg} / \mathrm{dL}$, hiperuricemia con ácido úrico en $8,57 \mathrm{mg} / \mathrm{dL}$ y elevación de los valores de creatinina sérica en $1,36 \mathrm{mg} / \mathrm{dL}$. Con todos los datos anteriores, se consideró un paciente en crisis adrenal con deterioro de función renal e hiperfosfatemia con hiperuricemia. Se inició el manejo inmediato con hidrocortisona y se mantuvo el soporte constante de líquidos y electrólitos, frente a lo cual mejoró la hipotensión, hiponatremia e hiperpotasemia. Se realizaron estudios imagenológicos con una TAC de abdomen contrastada en la que se evidenció engrosamiento marcado de ambas glándulas suprarrenales, principalmente la izquierda, con realce homogéneo del medio de contraste, pero con una lesión aparentemente focal de la glándula suprarrenal izquierda que medía $21 \mathrm{~mm}$. También se evidenciaron ganglios de las cadenas inguinales bilaterales ligeramente aumentados de tamaño (Figura 1).

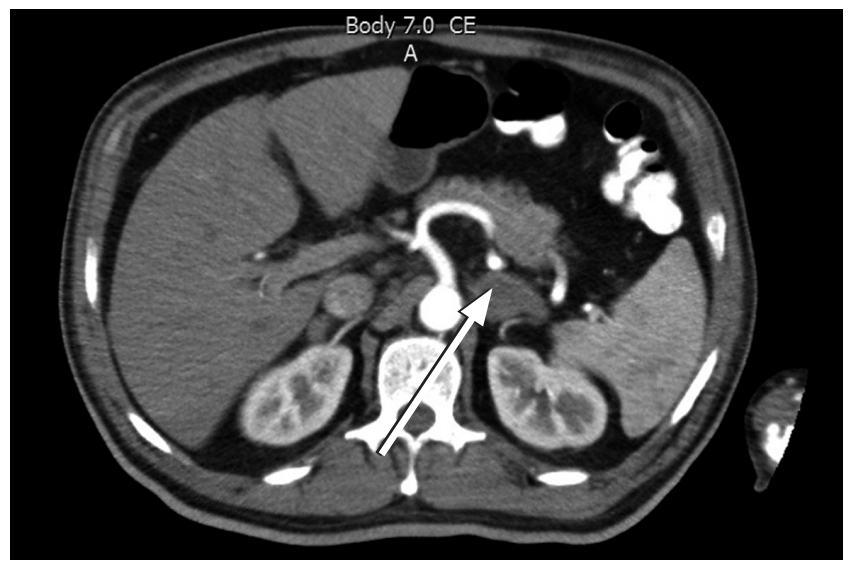

Figura 1. TAC de abdomen en la que se aprecia un aumento difuso de ambas glándulas suprarrenales, predominantemente la izquierda (flecha blanca).

El grupo tratante solicitó endosonografía para ver la posibilidad de toma de biopsia transgástrica de la lesión suprarrenal izquierda. Se realizó endosonografía pancreatobiliar con equipo lineal Pentax y procesador Hitachi, identificando en estación de la unión esofagogástrica una glándula suprarrenal izquierda aumentada de tamaño y con aspecto de masa heterogénea, pero predominantemente hipoecogénica sin una aparente lesión dominante, que llegó a medir hasta $25 \mathrm{~mm}$ de mayor dimensión.

Se realizó una biopsia de la masa suprarrenal izquierda guiada por endosonografía por punción con aguja número 
$22 \mathrm{G}$, con 7 pases y retirando el estilete, de tal forma que se obtuvo el material adecuado para su estudio.

No hubo complicaciones relacionadas con el procedimiento (Figura 2). El reporte de histopatología fue la presencia de necrosis e inflamación, y a la coloración de Gomori-Grocott se evidenció la presencia de levaduras multigemantes de tamaño variable compatibles con paracoccidioidomicosis. La tinción de Ziehl-Neelsen fue negativa para bacilos ácido-alcohol resistentes y no se evidenció malignidad en la muestra (Figuras 3 y 4). El paciente recibió manejo con anfotericina $B$ hasta completar el esquema y fue dado de alta por mejoría clínica evidente, con manejo ambulatorio con itraconazol y prednisona oral.

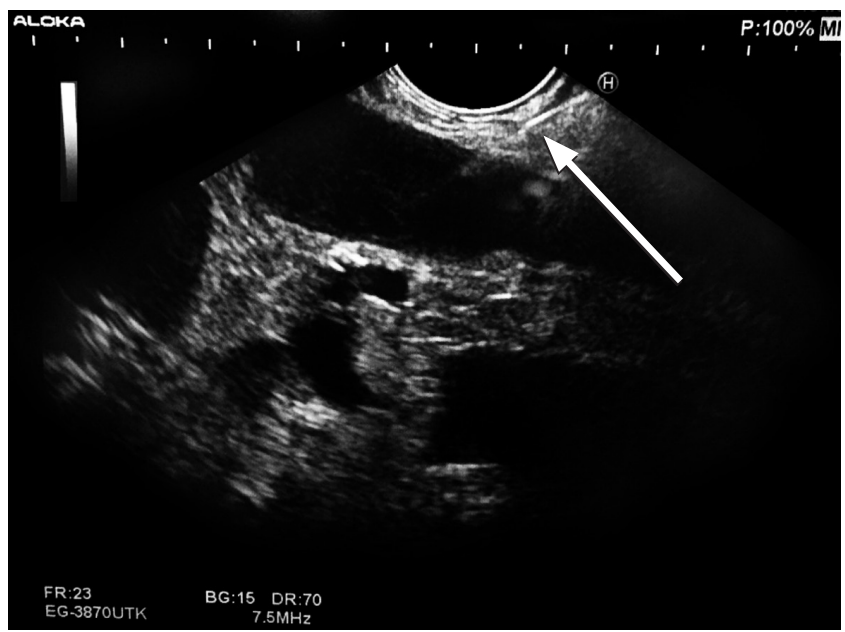

Figura 2. Endosonografía con visualización de la glándula suprarrenal izquierda aumentada de tamaño, de aspecto heterogéneo, hipoecogénica, con aspecto de masa. Biopsia por punción de glándula suprarrenal izquierda guiada por endosonografía (flecha blanca).

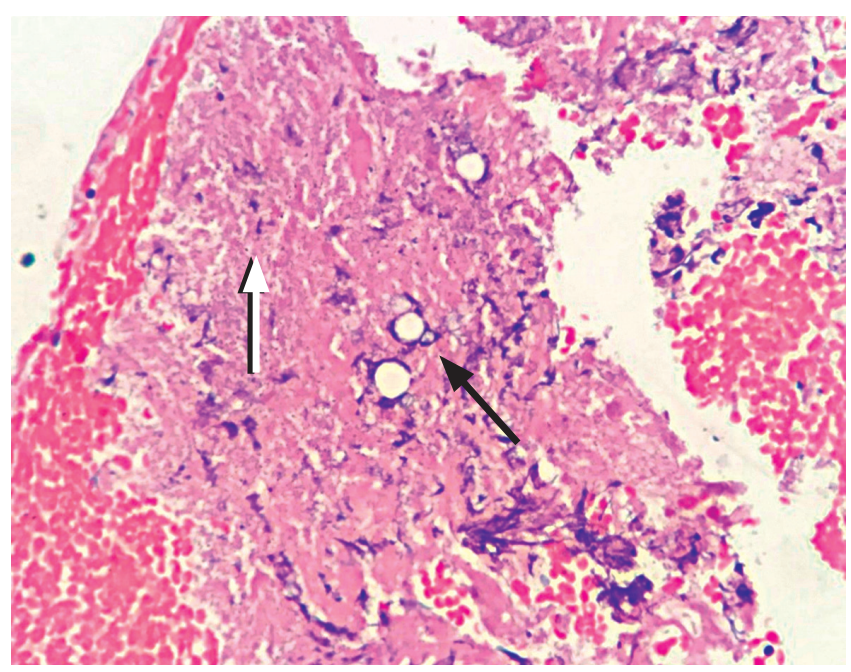

Figura 3. Visualización histológica con coloración de hematoxilinaeosina, tejido necrótico (flecha blanca) de glándula suprarrenal izquierda con presencia de levaduras multigemantes (flecha negra).

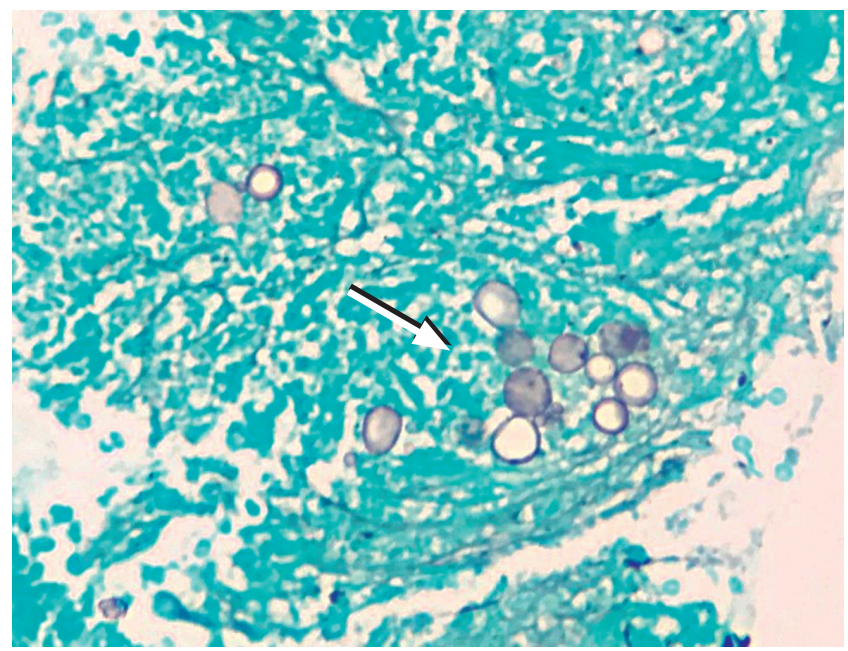

Figura 4. Histología, coloración de Gomori-Grocott. Evidencia de levaduras multigemantes compatibles con paracoccidioidomicosis (flecha blanca).

\section{DISCUSIÓN}

La paracoccidioidomicosis es una micosis sistémica prevalente en Latinoamérica, causada por el hongo dimórfico Paracoccidioides brasiliensis. El órgano que se afecta principalmente es el pulmón, pero puede verse comprometida la mucosa oral, sistema reticuloendotelial, piel, huesos y glándulas suprarrenales. En su presentación clínica se reconocen 2 formas: la aguda o subaguda (tipo juvenil), que afecta principalmente a niños y es de diseminación rápida afectando principalmente el sistema reticuloendotelial; y la crónica (tipo adulto), que corresponde a más del $90 \%$ de los casos e inicia como una infección pulmonar que se propaga a los ganglios linfáticos regionales $y$, dependiendo de la respuesta inmune, se puede eliminar, permanecer latente o activa (5).

Las glándulas suprarrenales se ven afectadas hasta en un $40 \%$ de los casos, pero hasta en $10 \%$ de los mismos el daño tisular es tan severo que las manifestaciones clínicas de la enfermedad corresponden a una insuficiencia suprarrenal $(3,5)$.

El diagnóstico definitivo se realiza con la visualización microscópica de células gigantes tipo Langhans y levaduras con gemación múltiple en patrón de timón de barco, con una mayor que tiene varias menores en gemación, en su periferia. Las tinciones del ácido peryódico de Schiff (PAS) y de plata permiten una mejor visualización de dicho hallazgo (6).

Cuando la forma de presentación de la paracoccidioidomicosis es con falla suprarrenal y alteración anatómica de las adrenales, el diagnóstico se logra con una biopsia de dichas glándulas (7).

Históricamente, los métodos de elección para la biopsia de las glándulas suprarrenales habían sido la ecografía transabdominal y la TAC con técnica percutánea. Sin embargo, dichos abordajes tienen tasas de muestras no diagnósticas 
hasta del $14 \%$ y se asocian con efectos adversos entre el $0,4 \%$ y $12 \%$, dentro de los que se incluyen neumotórax, sangrado, diseminación tumoral en el tracto de punción, abscesos adrenales y pancreatitis (8).

En la actualidad, se reconoce que la endosonografía logra la visualización de la glándula suprarrenal izquierda hasta en el $98 \%$ de los estudios y que las biopsias de glándula suprarrenal izquierda guiadas por endosonografía tienen un éxito técnico del $100 \%$, con mínimas y leves complicaciones asociadas, como sangrados menores (9). De igual forma, se considera una técnica para diagnóstico con biopsia de la glándula suprarrenal con un mejor perfil que las técnicas percutáneas, teniendo en cuenta especialmente que solo se atraviesa la pared gástrica para llegar al sitio de toma de la muestra. Incluso, se ha llegado a demostrar que, en pacientes que fueron sometidos a biopsia de glándula suprarrenal y no se les logró obtener muestra adecuada por abordaje percutáneo, o que incluso no se realizó el mismo por dificultades técnicas, el abordaje transgástrico guiado por endosonografía permitió una muestra adecuada de la que se logró el diagnóstico en todos los casos, como lo han reportado Puri y colaboradores (10).

Como consideración importante, se recomienda descartar la presencia de un feocromocitoma previo a la biopsia de glándula suprarrenal, para prevenir el advenimiento de una crisis hipertensiva a partir de una hemorragia en el sitio de la toma de la biopsia (11).

\section{CONCLUSIONES}

La paracoccidioidomicosis es una enfermedad exclusiva de algunos países de Latinoamérica donde se encuentra su patógeno causal; por tanto, los reportes de los casos presentados están limitados a ciertas áreas geográficas específicas. De igual forma, la afectación de las glándulas suprarrenales no se presenta en todos los casos y, por las alteraciones morfológicas que conlleva a nivel de dicho órgano, requiere un diagnóstico diferencial que se puede lograr de forma adecuada con biopsia. Con el paso del tiempo, se ha demostrado que las biopsias percutáneas de las glándulas suprarrenales conllevan un perfil de riesgo de complicaciones considerable, por lo que las biopsias guiadas por endosonografía han tomado un papel fundamental, teniendo en cuenta su adecuado perfil de rendimiento y seguridad.

Con la presentación de este caso, se amplía la posibilidad de diagnósticos diferenciales en la presencia de lesión de glándula suprarrenal y se debe tener en cuenta la posibilidad de paracoccidioidomicosis.
En la actualidad, no existen reportes en la literatura de paracoccidioidomicosis diagnosticada en la glándula suprarrenal por medio de biopsia guiada por endosonografía, por lo que el caso de este paciente es de gran importancia para el medio científico.

\section{REFERENCIAS}

1. Venkatachalapathy S, Nayar MK. Therapeutic endoscopic ultrasound. Frontline Gastroenterol. 2017;8(2):119-23. https://doi.org/10.1136/flgastro-2016-100774.

2. Bovio S, Cataldi A, Reimondo G, et al. Prevalence of adrenal incidentaloma in a contemporary computerized tomography series. J Endocrinol Invest. 2006;29(4):298-302. https://doi.org/10.1007/BF03344099.

3. Bodtger U, Vilmann P, Clementsen P, et al. Clinical impact of endoscopic ultrasound-fine needle aspiration of left adrenal masses in established or suspected lung cancer. J Thorac Oncol. 2009;4(12):1485-9. https://doi.org/10.1097/ JTO.0b013e3181b9e848.

4. Colaiacovo R, Ganc RL, Leone AC, et al. Diagnosis of left adrenal paracoccidioidomycosis by endoscopic ultrasound fine needle aspiration. Endoscopy. 2011;43 Suppl 2 UCTN:E236-7.

5. Pereira CM, Silva SR, Camargo P, et al. Addison's disease associated to paracocidioidomycosis. Braz J Oral Sci. 2004;3(9):475-8.

6. Farina M, Espinola C, Yamanaka W, et al. Paracoccidioidomicosis en la glándula suprarenal. Reporte de un caso. Rev Chil Cir. 2007;59(4):299-301. https://doi. org/10.4067/S0718-40262007000400010.

7. Agudelo CA, Muñoz C, Ramírez A, et al. Identification of Paracoccidioides brasiliensis in adrenal glands biopsies of two patients with paracoccidioidomycosis and adrenal insufficiency. Rev Inst Med Trop Sao Paulo. 2009;51(1):45-8. https://doi.org/10.1590/S0036-46652009000100008.

8. DeWitt JM. Endoscopic ultrasound-guided fine-needle aspiration of right adrenal masses: report of 2 cases. J Ultrasound Med. 2008;27(2):261-7. https://doi. org/10.7863/jum.2008.27.2.261.

9. Patil R, Ona MA, Papafragkakis C, et al. Endoscopic ultrasound-guided fine-needle aspiration in the diagnosis of adrenal lesions. Ann Gastroenterol. 2016;29(3):307-11. https://doi.org/10.20524/aog.2016.0047.

10. Puri R, Thandassery RB, Choudhary NS, et al. Endoscopic ultrasound-guided fine-needle aspiration of the adrenal glands: analysis of 21 patients. Clin Endosc. 2015;48(2):165-70. https://doi.org/10.5946/ce.2015.48.2.165.

11. Casola G, Nicolet V, vanSonnenberg E, et al. Unsuspected pheochromocytoma: risk of blood-pressure alterations during percutaneous adrenal biopsy. Radiology. 1986;159(3):733-5. https://doi.org/10.1148/radiology.159.3.3517958. 